\title{
Prediction of pathologic node-negative clinical stage IA lung adenocarcinoma for optimal candidates undergoing sublobar resection
}

\author{
Yasuhiro Tsutani, MD, PhD, ${ }^{\mathrm{a}}$ Yoshihiro Miyata, $\mathrm{MD}, \mathrm{PhD},{ }^{\mathrm{a}}$ Haruhiko Nakayama, $\mathrm{MD}, \mathrm{PhD},{ }^{\mathrm{b}}$ \\ Sakae Okumura, $\mathrm{MD}, \mathrm{PhD},{ }^{\mathrm{c}}$ Shuji Adachi, $\mathrm{MD}, \mathrm{PhD},{ }^{\mathrm{d}}$ Masahiro Yoshimura, $\mathrm{MD}, \mathrm{PhD},{ }^{\mathrm{e}}$ and \\ Morihito Okada, $\mathrm{MD}, \mathrm{PhD}^{\mathrm{a}}$
}

Objective: Patients with pathologic node-negative early lung cancer may be optimal candidates for sublobar resection. We aimed to identify predictors of pathologic lymph node involvement in clinical stage IA lung adenocarcinoma.

\begin{abstract}
Methods: The data from a multicenter database of 502 patients with completely resected clinical stage IA lung adenocarcinoma were retrospectively analyzed to determine the relationship between the lymph node metastasis status and tumor size on high-resolution computed tomography (HRCT) or maximum standardized uptake value (SUVmax) on [18F]-fluoro-2-deoxy-D-glucose positron emission tomography/computed tomography (FDGPET/CT). Revised SUVmax was used to correct interinstitutional discrepancies.

Results: In multivariate analyses, either a solid tumor size on HRCT $(P=.001)$ or an SUVmax on FDG-PET/CT $(P=.049)$ was an independent predictor of lymph node metastasis. The predictive criteria of pathologic nodenegative early lung cancer were a solid tumor size of less than $0.8 \mathrm{~cm}$ or an SUVmax of less than 1.5. Patients who met the predictive criteria of pathologic node-negative disease had less pathologic invasiveness, such as lymphatic, vascular, or pleural invasion $(P<.001)$, and better disease-free survival $(P<.0001)$ than those who did not, and $86(40.4 \%)$ of the 213 patients with T1b $(2-3 \mathrm{~cm})$ tumors met the predictive criteria.
\end{abstract}

Conclusions: Either a solid tumor size or an SUVmax was a significant independent predictor of nodal involvement in clinical stage IA lung adenocarcinoma. The pathologic node-negative status criteria of a solid tumor size of less than $0.8 \mathrm{~cm}$ on HRCT or an SUVmax of less than 1.5 on FDG-PET/CT may be helpful for avoiding systematic lymphadenectomy for clinical stage IA lung adenocarcinoma, even in cases of T1b $(2-3 \mathrm{~cm})$ tumor. (J Thorac Cardiovasc Surg 2012;144:1365-71)

Earn CME credits at

http://cme.ctsnetjournals.org

Recent advances in radiography, such as high-resolution computed tomography (HRCT) and the widespread practice of low-dose helical computed tomography (CT) for screening of tumors, have resulted in an increase in the early detection of smaller non-small cell lung cancer (NSCLC), especially adenocarcinoma. ${ }^{1-3}$ Several studies have

\footnotetext{
From the Department of Surgical Oncology, ${ }^{\text {a }}$ Hiroshima University, Hiroshima; the Department of Thoracic Surgery, ${ }^{\mathrm{b}}$ Kanagawa Cancer Center, Yokohama; the Department of Thoracic Surgery, ${ }^{\mathrm{c}}$ Cancer Institute Hospital, Tokyo; and the Departments of Radiology ${ }^{\mathrm{d}}$ and Thoracic Surgery, ${ }^{\mathrm{e}}$ Hyogo Cancer Center, Akashi, Japan. Disclosures: Authors have nothing to disclose with regard to commercial support.

Received for publication Feb 16, 2012; revisions received June 7, 2012; accepted for publication July 10, 2012; available ahead of print Aug 10, 2012.

Address for reprints: Morihito Okada, MD, PhD, Department of Surgical Oncology,

Research Institute for Radiation Biology and Medicine, Hiroshima University, 1-2-

3-Kasumi, Minami-ku, Hiroshima City, Hiroshima 734-0037, Japan (E-mail:

morihito@hiroshima-u.ac.jp).

$0022-5223 / \$ 36.00$

Copyright (C) 2012 by The American Association for Thoracic Surgery

http://dx.doi.org/10.1016/j.jtcvs.2012.07.012
}

reported that the survival is similar in patients with small peripheral NSCLC between those treated with segmentectomy and those treated with lobectomy. ${ }^{1,4-8}$ In determining the indications for sublobar resection, prediction of the pathologic node-negative (pN0) status is important. ${ }^{4-8} \mathrm{We}^{9}$ previously reported that solid tumor size on HRCT and maximum standardized uptake value (SUVmax) on [18F]-fluoro-2-deoxy-D-glucose positron emission tomography/computed tomography (FDG-PET/ $\mathrm{CT}$ ) were useful for predicting pathologic invasiveness or prognosis in clinical stage IA lung adenocarcinoma. We hypothesized that solid tumor size on HRCT and SUVmax on FDG-PET/CT had a predictive value for lymph node metastasis. Therefore, we retrospectively investigated the preoperative predictive value of solid tumor size and SUVmax for pNO status to select optimal candidates for sublobar resection in clinical stage IA lung adenocarcinoma. The primary end point of this study was lymph node involvement.

\section{PATIENTS AND METHODS \\ Patients}

We enrolled 502 patients with clinical T1 N0 M0 stage IA lung adenocarcinoma from 4 institutions in Japan (Hiroshima University, Kanagawa 


\section{Abbreviations and Acronyms \\ CT = computed tomography \\ DFS = disease-free survival \\ FDG $=[18 \mathrm{~F}]$-fluoro-2-deoxy-D-glucose \\ GGO = ground-glass opacity \\ HRCT = high-resolution computed tomography \\ NSCLC $=$ non-small cell lung cancer \\ PET = positron emission tomography \\ SUVmax $=$ maximum standardized uptake value}

Cancer Center, Cancer Institute Hospital, and Hyogo Cancer Center) between August 1, 2005, and December 31, 2009, to evaluate the significance of FDG-PET/CT. ${ }^{10}$ The data from all 502 patients from this multicenter database were retrospectively analyzed in the present study. HRCT and FDGPET/CT followed by curative R0 resection were performed for all patients who were staged according to the TNM Classification of Malignant Tumours, 7 th edition. ${ }^{11}$ Mediastinoscopy or endobronchial ultrasonography was not routinely performed because all patients received preoperative HRCT and FDG-PET/CT; HRCT revealed no swelling of mediastinal or hilar lymph nodes and FDG-PET showed no accumulation in those lymph nodes. Sublobar resection was allowed in cases of complete removal of the disease using a procedure for a peripheral T1a N0 M0 tumor. Wedge resection without lymph node assessment was allowed for a pure ground-glass opacity (GGO) tumor, which was regarded as a nodenegative noninvasive tumor in a prospective study. ${ }^{12}$ Segmentectomy with hilar and mediastinal lymph node dissection was allowed for a GGO-mixed tumor. If lymph node involvement was evident by intraoperative frozen section of any lymph node, the procedure was converted to standard lobectomy. All of the other patients underwent standard lobectomy. The inclusion criteria included preoperative staging determined by HRCT and FDG-PET/CT, curative surgery without neoadjuvant chemotherapy or radiotherapy, and a definitive histopathologic diagnosis of lung adenocarcinoma. Patients with incompletely resected tumors (R1 or R2) and those with multiple tumors or previous lung surgery were excluded from the database. This multicenter study was approved by the institutional review boards of the participating institutions, all of which waived the requirement for informed consent from individual patients for this retrospective review of the prospective database.

Table 1 summarizes the characteristics of the 502 patients enrolled in this study. The mean follow-up period after surgery was $19.8 \pm 12.2$ months, during which disease recurred in $29(5.8 \%)$ patients. There were $9(1.8 \%)$ local recurrences, including mediastinal lymph node metastasis, $3(0.6 \%)$ local and distant recurrences, and $17(3.4 \%)$ distant recurrences. The median whole tumor and solid tumor sizes on HRCT were $2.0 \mathrm{~cm}$ and $1.2 \mathrm{~cm}$, respectively. The median SUVmax was 2.0. Lymphatic, vascular, and pleural invasion was evident in $76(15.1 \%), 92(18.3 \%)$, and 56 $(11.2 \%)$ patients, respectively, and lymph nodes were involved in 38 $(7.8 \%)$.

\section{High-Resolution Computed Tomography}

Sixteen-row multidetector CT was used to obtain chest images independent of subsequent FDG-PET/CT examinations. For high-resolution images of the tumors, the following parameters were used: $120 \mathrm{kVp}, 200$ $\mathrm{mA}, 1$ to 2 -mm section thickness, $512 \times 512$-pixel resolution, 0.5 to 1.0 second scanning time, a high-spatial reconstruction algorithm with a 20-cm field of view, and mediastinal (level, $40 \mathrm{HU}$; width, $400 \mathrm{HU}$ ) and lung (level, $-600 \mathrm{HU}$; width, $1600 \mathrm{HU}$ ) window settings. GGO was defined as a misty increase in lung attenuation that did not obscure underlying vascular markings. We defined solid tumor size as the maximum dimension of the solid component of the lung windows, excluding GGO. ${ }^{11} \mathrm{CT}$ scans were reviewed and tumor sizes determined by radiologists from each institution for the purpose of this study.

\section{FDG-PET/CT}

Patients were instructed to fast for more than 4 hours before intravenous injection of 74 to $370 \mathrm{MBq} F D G$ and then to relax for at least 1 hour before FDG-PET/CT scanning. Blood glucose levels were calculated before the tracer injection to confirm a level of less than $150 \mathrm{mg} / \mathrm{dL} .{ }^{12}$ Patients with blood glucose levels of $150 \mathrm{mg} / \mathrm{dL}$ or more were excluded from PET/CT acquisition. For imaging, Discovery ST (GE Healthcare, Little Chalfont, United Kingdom), Aquiduo (Toshiba Medical Systems Corporation, Tochigi, Japan), or Biograph Sensation16 (Siemens Healthcare, Erlangen, Germany) integrated 3-dimensional PET/CT scanners were used. Lowdose nonenhanced CT images of 2 to 4-mm section thickness for attenuation correction and localization of lesions identified by PET were obtained from the head to the pelvic floor of each patient by following a standard protocol. Immediately after CT, PET covered the identical axial field of view for 2 to 4 minutes per table position, depending on the condition of the patient and the scanner performance. An iterative algorithm with CTderived attenuation correction was used to reconstruct all PET images with a $50-\mathrm{cm}$ field of view. An anthropomorphic body phantom (NEMA NU2-2001; Data Spectrum Corp, Hillsborough, NC) was used to minimize variations in SUVs among the institutions. A calibration factor was analyzed by dividing the actual SUV by the gauged mean SUV in the phantom background to decrease interinstitutional SUV inconsistencies; the final SUV used here is referred to as the revised SUVmax. ${ }^{13,14}$ The adjustment of interinstitutional variations in SUV narrowed the range from 0.89 to 1.24 to 0.97 to 1.18 when the SUVmax ratio was expressed as the SUVmax of each institute relative to the SUVmax of the control institute. The original SUVmax values were determined by radiologists from each institution for the purpose of this study.

\section{Follow-up Evaluation}

All patients who underwent lung resection were followed up from the day of surgery. Postoperative follow-up procedures, including a physical examination and chest radiograph every 3 months and chest and abdominal CT examinations every 6 months, were performed for the first 2 years. Afterward, a physical examination and chest radiograph were performed every 6 months, and a chest CT examination was performed every year.

\section{Statistical Analysis}

Data are presented as numbers (percents) or the median unless otherwise stated. Multiple logistic regression analyses were performed to determine the independent continuous variables related to whole tumor size, solid tumor size, SUVmax, and carcinoembryonic antigen for prediction of pathologic lymph node metastasis. In addition, receiver operating characteristic curves of solid tumor size and SUVmax were used for determining the criteria of $\mathrm{pNO}$. The $\chi^{2}$ test for categorical variables was used to compare frequencies and Fisher's exact test was applied to small samples. Disease-free survival (DFS) was defined as the time from the date of surgery until the first event (relapse or death from any cause) or last followup. The Kaplan-Meier method was used to analyze the duration of DFS, and the log-rank test was used to assess differences in DFS. SPSS software (version 10.5; SPSS Inc, Chicago, Ill) was used to statistically analyze the data.

\section{RESULTS}

Possible predictors of lymph node metastasis were investigated (Table 2). Multivariate analysis showed that solid tumor size or SUVmax, but not whole tumor size and carcinoembryonic antigen, were independent predictors of lymph node metastasis. 
TABLE 1. Patient characteristics

\begin{tabular}{lc}
\hline \multicolumn{1}{c}{ Variable } & $\mathbf{n}=\mathbf{5 0 2}$ \\
\hline Age (y, range) & $66(31-86)$ \\
Gender & \\
$\quad$ Male & $223(44.4 \%)$ \\
Female & $279(55.6 \%)$ \\
Whole tumor size (cm, range) & $2.0(0.6-3.0)$ \\
Solid tumor size (cm, range) & $1.2(0-3.0)$ \\
SUVmax (range) & $2.0(0-27.7)$ \\
CEA (ng/mL, range) & $2.4(0-113.8)$ \\
Procedure & \\
Lobar resection & $320(63.7 \%)$ \\
$\quad$ Sublobar resection (wedge + segmentectomy) & $182(36.3 \%)$ \\
Lymphatic invasion & \\
$\quad$ Negative & $426(84.9 \%)$ \\
Positive & $76(15.1 \%)$ \\
Vascular invasion & \\
$\quad$ Negative & $410(81.7 \%)$ \\
Positive & $92(18.3 \%)$ \\
Pleural invasion & \\
$\quad$ Negative & $446(88.8 \%)$ \\
Positive & $56(11.2 \%)$ \\
Lymph node metastasis & \\
Negative & $464(92.4 \%)$ \\
Positive & $38(7.6 \%)$ \\
\hline SUVmax, Maximum standardized uptake value; $C E A$, carcinoembryonic antigen.
\end{tabular}

Figure 1 shows the receiver operating characteristic area under the curve values for solid tumor size and SUVmax used in determining the criteria of pNO. When using solid tumor size and SUVmax for predicting pNO, patients with a solid tumor size of less than $0.8 \mathrm{~cm}$ or an SUVmax of less than 1.5 were observed to have no lymph node metastasis. We defined the predictive criteria of $\mathrm{pN} 0$ as a solid tumor size of less than $0.8 \mathrm{~cm}$ or an SUVmax of less than 1.5.

Table 3 shows the number of patients who met the criteria of $\mathrm{pNO}$ as indicated by solid tumor size, SUVmax, and a combination of the two. Using this combination, the number of patients expected to have pN0 was $169(58.5 \%)$ of 289 for clinical T1a $(\leq 2 \mathrm{~cm})$ and $86(40.4 \%)$ of 213 for clinical T1b $(2-3 \mathrm{~cm})$ tumors. Figure 2 shows examples of tumors larger than $2 \mathrm{~cm}$ that met the predictive criteria of $\mathrm{pN} 0$.

TABLE 2. Multivariate analysis of possible predictors of lymph node metastasis

\begin{tabular}{lll}
\hline \multicolumn{1}{c}{ Valuables } & OR $(\mathbf{9 5} \% \mathbf{C I})$ & $\boldsymbol{P}$ \\
\hline Model 1 & & \\
$\quad$ Whole tumor size $(\mathrm{cm})$ & $1.42(0.76-2.66)$ & .28 \\
SUVmax & $1.09(1.00-1.29)$ & .049 \\
CEA (ng/mL) & $1.04(0.99-1.08)$ & .097 \\
Model 2 & & \\
Solid tumor size $(\mathrm{cm})$ & $2.44(1.49-4.00)$ & $<.001$ \\
SUVmax & $1.04(0.93-1.16)$ & .47 \\
CEA $(\mathrm{ng} / \mathrm{mL})$ & $1.03(0.99-1.07)$ & .14 \\
\hline
\end{tabular}

OR, Odds ratio; $C I$, confidence interval; SUVmax, maximum standardized uptake value; $C E A$, carcinoembryonic antigen.
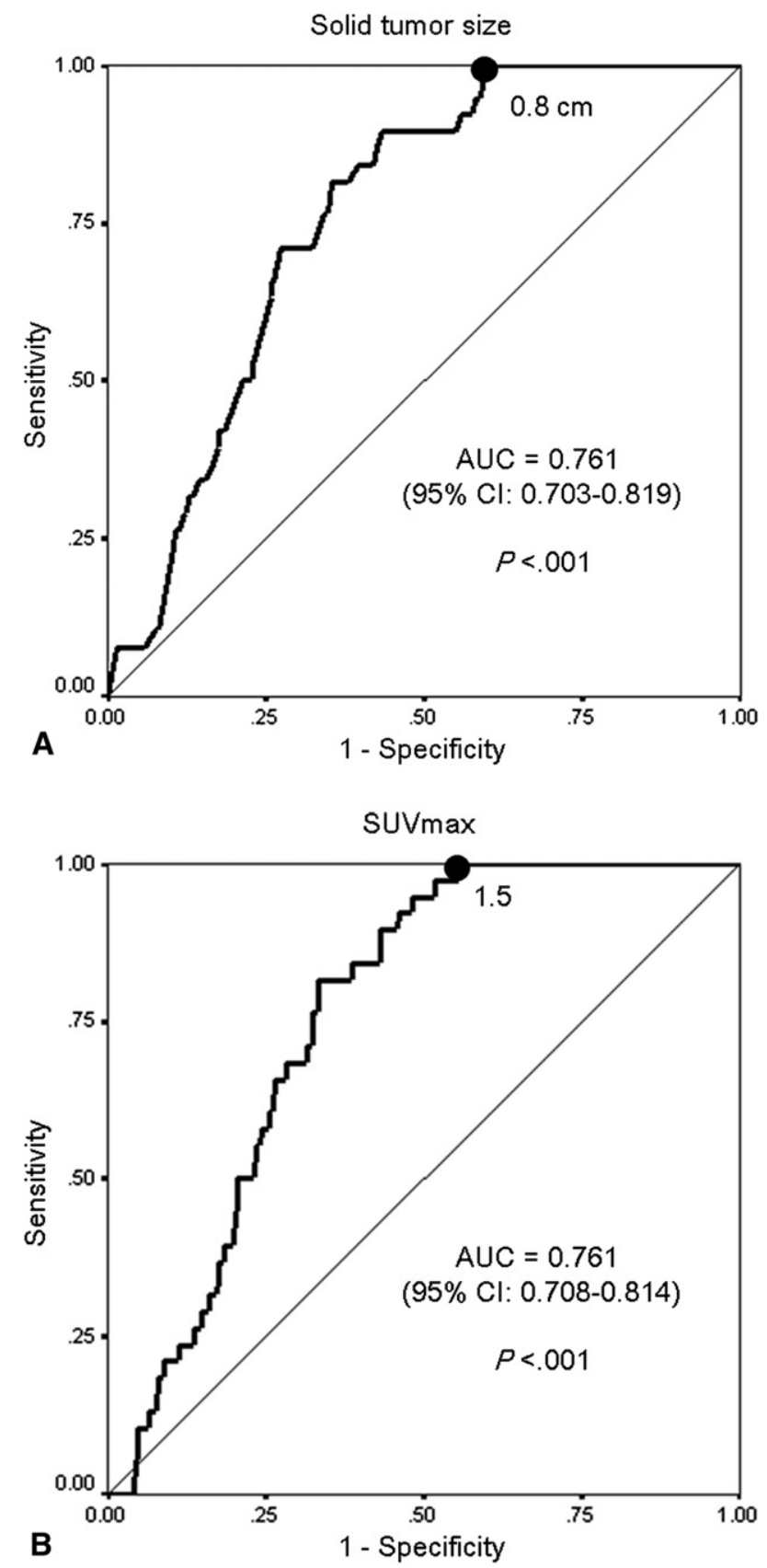

FIGURE 1. Receiver operating characteristic area under the curve (AUC) for detecting lymph node metastasis for solid tumor size (A) and maximum standardized uptake value (SUVmax) (B). Solid tumor size: AUC, 0.761 (95\% confidence interval $[C I], 0.703-0.819 ; P<.001)$. SUVmax: AUC, 0.761 (95\% CI, 0.708-0.814; $P<.001)$.

A significant difference in DFS for all T1 tumors was identified between patients with a solid tumor size of less than $0.8 \mathrm{~cm}$ or an SUVmax of less than $1.5(\mathrm{n}=255$; 3 -year DFS rate, $100 \%$ ) and those with a solid tumor size of $0.8 \mathrm{~cm}$ or more and an SUVmax of 1.5 or more $(\mathrm{n}=247 ; 3$-year DFS rate, $81.8 \% ; P<.001$ ) (Figure 3, $A)$. Even when cases were categorized into cT1a and 
TABLE 3. Number of patients without nodal metastasis according to solid tumor size, SUVmax, and a combination of the two

\begin{tabular}{lccc}
\hline & $\begin{array}{c}\text { cT1 } \\
(\mathbf{n}=\mathbf{5 0 2})\end{array}$ & $\begin{array}{c}\text { cT1a } \\
(\mathbf{n}=\mathbf{2 8 9})\end{array}$ & $\begin{array}{c}\text { cT1b } \\
(\mathbf{n}=\mathbf{2 1 3})\end{array}$ \\
\hline Solid tumor size $<0.8 \mathrm{~cm}$ & $187(37.3 \%)$ & $131(45.3 \%)$ & $56(26.3 \%)$ \\
SUVmax $<1.5$ & $206(41.0 \%)$ & $138(47.8 \%)$ & $68(31.9 \%)$ \\
$\begin{array}{l}\text { Solid tumor size }<0.8 \mathrm{~cm} \\
\quad \text { or SUVmax }<1.5\end{array}$ & $255(50.8 \%)$ & $169(58.5 \%)$ & $86(40.4 \%)$ \\
\hline SUVmax Maximum standardized uptake value. & & \\
\hline
\end{tabular}

cT1b, significant differences in DFS were observed between patients with a solid tumor size of less than $0.8 \mathrm{~cm}$ or an SUVmax of less than 1.5 and the remainder (Figure 3, $B$ and $C$ ). Moreover, a significant difference in DFS was observed between patients with T1a tumor with a solid tumor size of $0.8 \mathrm{~cm}$ or more and an SUVmax of 1.5 or more and those with T1b tumor with a solid tumor size of less than $0.8 \mathrm{~cm}$ or an SUVmax of less than 1.5 $(P=.0015$; Figure $3, D)$.

Significant differences in pathologic invasiveness (lymphatic, vascular, or pleural invasion) were identified between patients with a solid tumor size of less than $0.8 \mathrm{~cm}$ or an SUVmax of less than 1.5 and those with a solid tumor size of $0.8 \mathrm{~cm}$ or more and an SUVmax of 1.5 or more (Table 4); a few patients who met the predictive criteria of

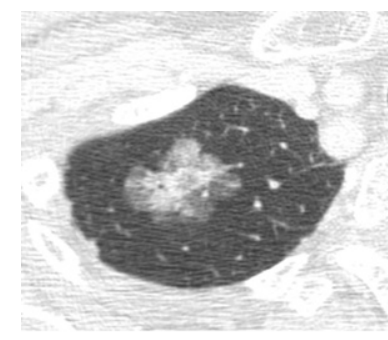

Whole tumor size $=2.8 \mathrm{~cm}$ A Solid tumor size $=0.7 \mathrm{~cm}$

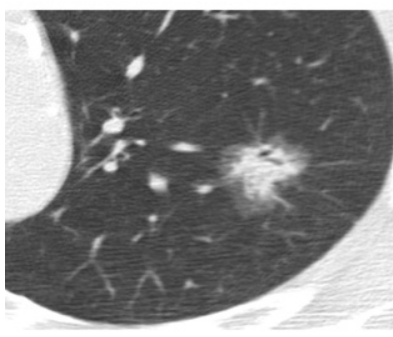

Whole tumor size $=2.4 \mathrm{~cm}$

B Solid tumor size $=1.5 \mathrm{~cm}$

FIGURE 2. Examples of tumors larger than $2 \mathrm{~cm}$ that met the predictive criteria of pathologic node negative. A, Whole tumor size, $2.8 \mathrm{~cm}$; solid tumor size, $0.7 \mathrm{~cm}$; maximum standardized uptake value (SUVmax), 1.8. This tumor can be treated with sublobar resection, such as right apical segmentectomy. B, Whole tumor size, $2.6 \mathrm{~cm}$; solid tumor size, $1.7 \mathrm{~cm}$; SUVmax, 0.9 . This tumor can be treated with sublobar resection, such as left apicoposterior segmentectomy.
pN0 had pathologic invasiveness $(9 / 255,3.5 \%)$. Even in the cases categorized as cT1a or cT1b, significant differences in pathologic invasiveness were observed between the patients with a solid tumor size of less than $0.8 \mathrm{~cm}$ or an SUVmax of less than 1.5 and those with a solid tumor size of $0.8 \mathrm{~cm}$ or more and an SUVmax of 1.5 or more (Table 4).

\section{DISCUSSION}

In 1995, the Lung Cancer Study Group conducted a prospective randomized controlled trial comparing limited resection with lobectomy for clinical T1 N0 M0 NSCLC and concluded that the former resulted in inferior local recurrence and survival. ${ }^{15}$ On the other hand, several reports have described how survival was similar between patients treated with segmentectomy and those treated with lobectomy. ${ }^{1,4-8}$ Determining the indications for sublobar resection requires that intraoperative frozen sections be examined for all hilar and lobe-specific mediastinal lymph nodes to confirm the intraoperative $\mathrm{N}$ staging as $\mathrm{N0}{ }^{1,4-7}$ However, intraoperative examination of many lymph nodes is difficult for thoracic surgeons and pathologists in the clinical setting. If $\mathrm{pNO}$ can be predicted from preoperative information, sublobar resection without strict intraoperative lymph node assessment can be performed in patients with clinical stage IA NSCLC.

The present study demonstrated the value of using solid tumor size with HRCT and SUVmax on PET/CT to predict the status of nodal involvement in clinical stage IA lung adenocarcinoma. $\mathrm{We}^{9}$ reported the usefulness of solid tumor size defined as the maximum dimension of the solid component, excluding GGO, on HRCT compared with that of whole tumor size for predicting pathologic invasiveness of tumors or prognosis in clinical stage IA lung adenocarcinoma. In that study, solid tumor size showed a higher predictive value for pathologic invasiveness than did whole tumor size, and it was an independent prognostic factor for DFS. We $\mathrm{e}^{9,10,14,16,17}$ also reported the usefulness of SUVmax to predict the malignancy grade of tumors with regard to pathologic invasiveness in lung adenocarcinoma. Solid tumor size and SUVmax were useful in predicting both pathologic nodal status and pathologic tumor invasiveness in the current study. Moreover, we found that patients with a solid tumor size of less than $0.8 \mathrm{~cm}$ or an SUVmax of less than 1.5 had pN0. There were significant differences in DFS between patients who met the predictive criteria of $\mathrm{pNO}$ and those who did not. Furthermore, the incidence of pathologic invasiveness in patients who met the predictive criteria of $\mathrm{pNO}$ was very low. These findings indicate that the predictive criteria of pN0 were reasonable for choosing a treatment strategy in clinical stage IA lung adenocarcinoma.

Interestingly, $40.4 \%$ of tumors larger than $2 \mathrm{~cm}$ and $58.5 \%$ of those $2 \mathrm{~cm}$ or smaller met the predictive criteria 


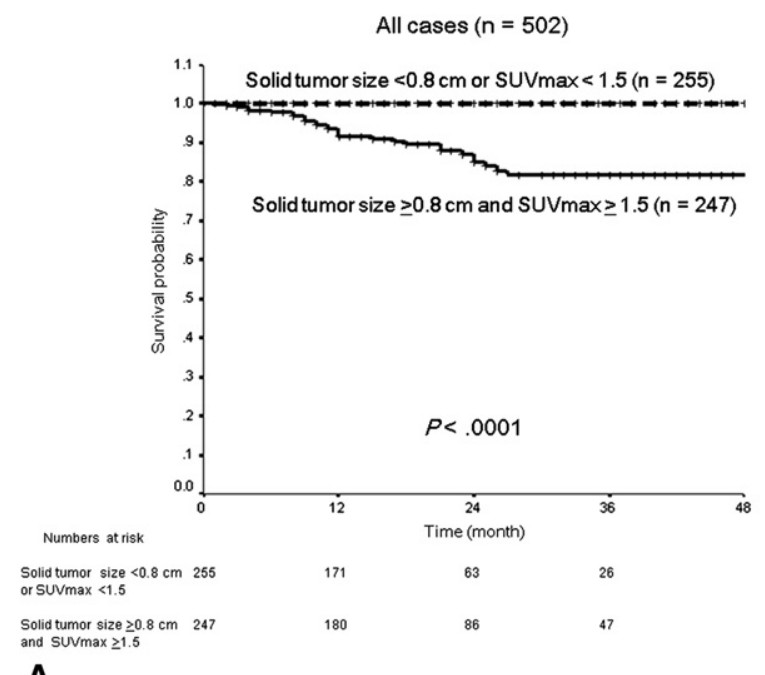

A

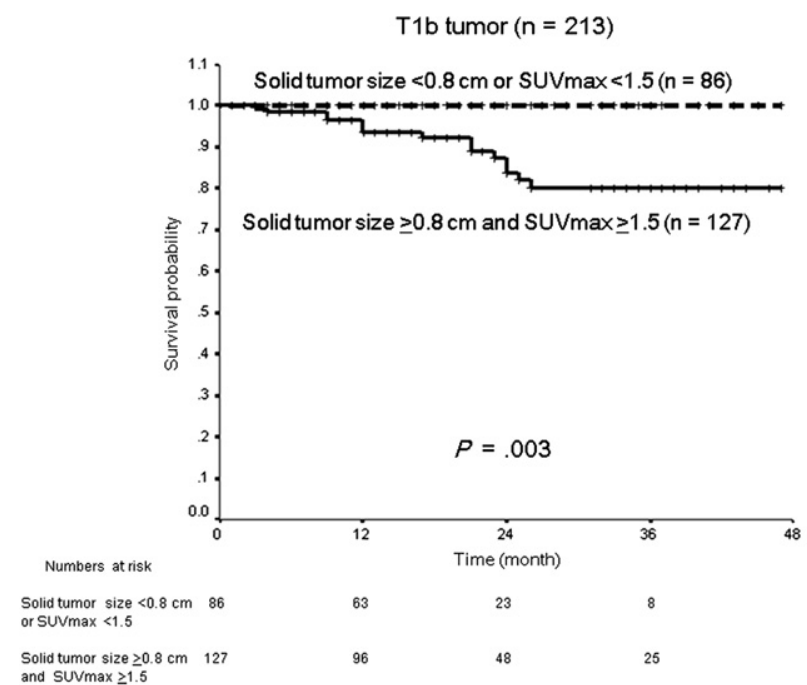

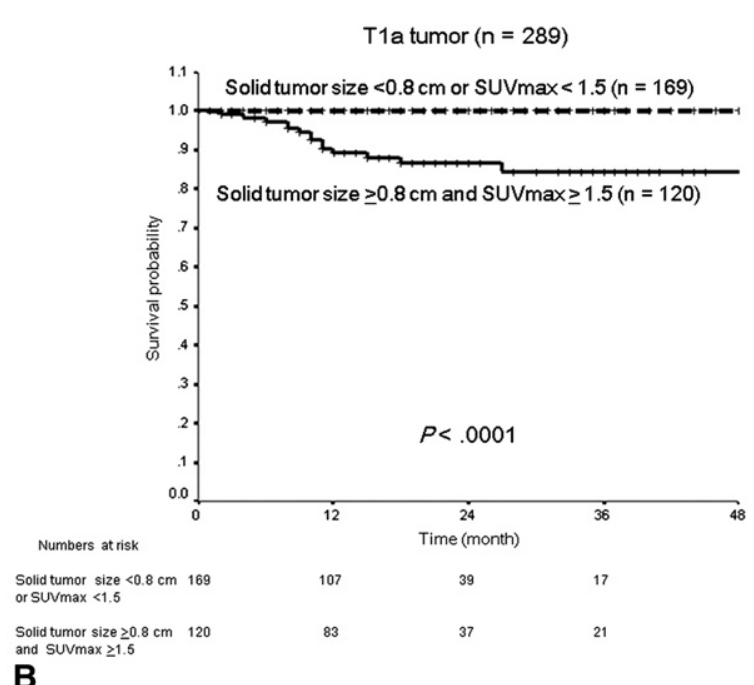

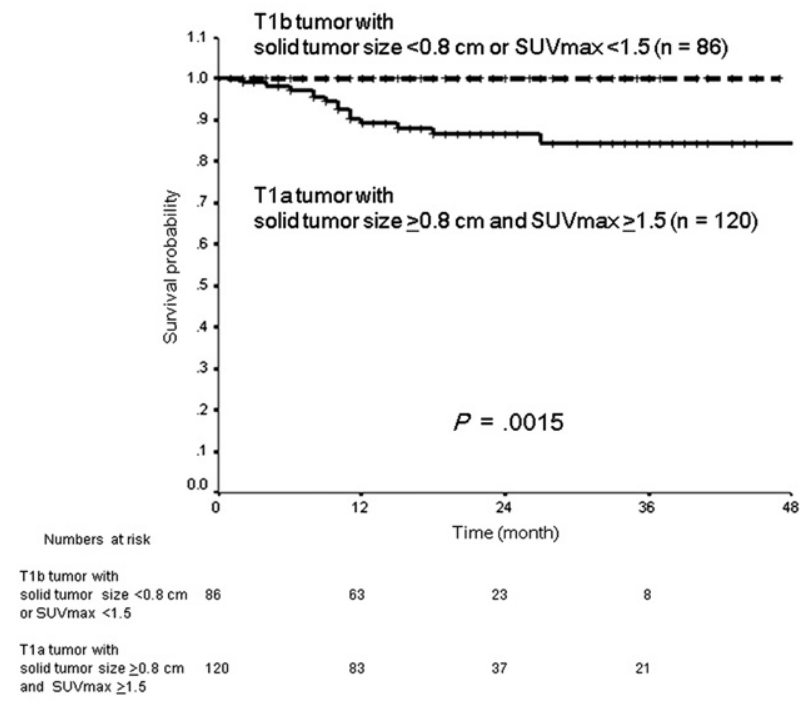

D

FIGURE 3. Disease-free survival $(D F S)$ curves of patients according to the predictive criteria of pathologic node negative using solid tumor size and maximum standardized uptake value (SUVmax). A, Three-year DFS rates of $100 \%$ and $81.8 \%$ for patients with a solid tumor size of less than $0.8 \mathrm{~cm}$ or an SUVmax of less than 1.5 and those with a solid tumor size of $0.8 \mathrm{~cm}$ or more and an SUVmax of 1.5 , respectively $(P<.0001)$ in all T1 tumors. B, Three-year DFS rate of $100 \%$ and $84.2 \%$ for patients with a solid tumor size of less than $0.8 \mathrm{~cm}$ or an SUVmax of less than 1.5 and those with a solid tumor size of $0.8 \mathrm{~cm}$ or more and an SUVmax of 1.5 or more, respectively $(P<.0001)$ in T1a $(\leq 2.0 \mathrm{~cm})$ tumors. C, Three-year DFS rate of $100 \%$ and $80.1 \%$ for patients with a solid tumor size of less than $0.8 \mathrm{~cm}$ or an SUVmax of less than 1.5 and those with a solid tumor size of $0.8 \mathrm{~cm}$ or more and an SUVmax of 1.5 or more, respectively $(P=.003)$ in T1b $(2-3 \mathrm{~cm})$ tumors. D, Three-year DFS rate of $100 \%$ and $84.2 \%$ for patients with a solid tumor size of less than $0.8 \mathrm{~cm}$ or an SUVmax of less than $1.5 \mathrm{in} \mathrm{T1b}$ tumors and those with a solid tumor size of $0.8 \mathrm{~cm}$ or more and an SUVmax of 1.5 or more in T1a tumors, respectively $(P=.0015)$.

of pNO. Most previous studies that showed favorable results for segmentectomy set its indication at T1 N0 M0 NSCLC of $2 \mathrm{~cm}$ or less. ${ }^{1,4-6}$ The ongoing clinical trials comparing surgical results between lobectomy and sublobar resection conducted by the Cancer and Leukemia Group B (CALGB 140503) and by the Japan Clinical Oncology Group/West Japan Oncology Group (JCOG0802/ WJOG4607L) also set a T1 N0 M0 NSCLC criterion of 2 $\mathrm{cm}$ or smaller for the study subjects. ${ }^{18}$ However, approximately $40 \%$ of patients with T1 N0 M0 lung adenocarcinoma of more than $2 \mathrm{~cm}$ might be candidates for sublobar resection according to the present study because such patients can be predicted as $\mathrm{pN} 0$ preoperatively. In fact, no recurrence was seen in patients with $\mathrm{T} 1 \mathrm{~b}$ tumor who met the predictive criteria, regardless of the surgical procedure. Furthermore, the frequency of pathologic invasiveness of $\mathrm{T} 1 \mathrm{~b}$ tumors that met the criteria of $\mathrm{pN} 0$ was very low. However, adequate surgical margins are required when performing 
TABLE 4. Comparison of pathologic invasiveness of tumors and lymph node involvement between patients who met the criteria of pathologic node negative and those who did not

\begin{tabular}{|c|c|c|c|}
\hline $\begin{array}{l}\text { All tumors } \\
(n=502)\end{array}$ & $\begin{array}{c}\text { Solid tumor size }<0.8 \mathrm{~cm} \text { or } \\
\text { SUVmax }<1.5(n=255)\end{array}$ & $\begin{array}{c}\text { Solid tumor size } \geq 0.8 \mathrm{~cm} \\
\text { and } S U V m a x \geq 1.5(n=247)\end{array}$ & $P$ \\
\hline LY & $4(1.6 \%)$ & $73(29.6 \%)$ & $<.001$ \\
\hline V & $4(1.6 \%)$ & $89(36.0 \%)$ & $<.001$ \\
\hline PL & $3(1.2 \%)$ & $55(22.3 \%)$ & $<.001$ \\
\hline LY or V or PL & $9(3.5 \%)$ & $131(53.0 \%)$ & $<.001$ \\
\hline $\mathrm{N}$ & $0(0 \%)$ & $38(15.4 \%)$ & $<.001$ \\
\hline $\begin{array}{c}\text { T1a tumors } \\
(\mathbf{n}=\mathbf{2 8 9}) \\
\end{array}$ & $\begin{array}{c}\text { Solid tumor size }<0.8 \mathrm{~cm} \text { or } \\
\text { SUVmax }<1.5(n=169)\end{array}$ & $\begin{array}{c}\text { Solid tumor size } \geq 0.8 \mathrm{~cm} \\
\text { and } S U V m a x \geq 1.5(n=120)\end{array}$ & $P$ \\
\hline LY & $3(1.8 \%)$ & $34(28.3 \%)$ & $<.001$ \\
\hline V & $4(2.4 \%)$ & $33(27.5 \%)$ & $<.001$ \\
\hline PL & $2(1.2 \%)$ & $24(20.0 \%)$ & $<.001$ \\
\hline LY or V or PL & $7(4.1 \%)$ & $59(49.2 \%)$ & $<.001$ \\
\hline $\mathrm{N}$ & $0(0 \%)$ & $16(13.3 \%)$ & $<.001$ \\
\hline $\begin{array}{l}\text { T1b tumors } \\
(\mathrm{n}=\mathbf{2 1 3}) \\
\end{array}$ & $\begin{array}{c}\text { Solid tumor size }<0.8 \mathrm{~cm} \text { or } \\
\text { SUVmax }<1.5(n=86) \\
\end{array}$ & $\begin{array}{c}\text { Solid tumor size } \geq 0.8 \mathrm{~cm} \\
\text { and } S U V m a x \geq 1.5(n=127)\end{array}$ & $P$ \\
\hline LY & $1(1.2 \%)$ & $39(30.7 \%)$ & $<.001$ \\
\hline V & $0(0 \%)$ & $56(44.1 \%)$ & $<.001$ \\
\hline PL & $1(1.2 \%)$ & $31(24.4 \%)$ & $<.001$ \\
\hline LY or V or PL & $2(2.3 \%)$ & $72(56.7 \%)$ & $<.001$ \\
\hline $\mathrm{N}$ & $0(0 \%)$ & $22(17.3 \%)$ & $<.001$ \\
\hline
\end{tabular}

SUVmax, Maximum standardized uptake value; $L Y$, lymphatic invasion; $V$, vascular invasion; $P L$, pleural invasion.

sublobar resection in such patients. To achieve complete resection with adequate surgical margins, we recommend segmentectomy, not wedge resection, for T1b tumors that meet the criteria of $\mathrm{pN} 0$. To provide an adequate surgical margin for T1b tumors by wedge resection is difficult. Local recurrence must be avoided. When performing lobar resection for tumors that meet the predictive criteria of $\mathrm{pN} 0$, systematic lymph node dissection such as mediastinal lymph node dissection is not always needed. On the other hand, when performing sublobar resection in patients with tumors of 2 $\mathrm{cm}$ or less, wedge resection and segmentectomy with ample surgical margins can be permitted. The extent of resection can be determined according to the tumor size and location. Lobe-specific lymph node dissection or sampling can be allowed for tumors that meet the criteria of $\mathrm{pN} 0$. Systematic lymph node dissection is not always needed for these tumors. The use of solid tumor size and SUVmax allows many more patients to be identified, who may benefit from the maintenance of lung function that results from selecting sublobar resection rather than can be identified simply on the basis of peripheral small nodules of $2 \mathrm{~cm}$ or less. The proportion of patients with lung adenocarcinoma of 2 $\mathrm{cm}$ or less who did not meet the predictive criteria of $\mathrm{pN} 0$ was approximately $40 \%$. There are some risks of lymph node metastasis in small lung adenocarcinoma that do not meet the predictive criteria of $\mathrm{pN} 0$. When sublobar resection is performed in such cases, intraoperative lymph node assessment, such as examination of frozen sections, is mandatory, and wedge resection cannot be recommended because such a procedure does not allow an approach to the hilar lymph nodes for assessment. If lymph node metastasis is intraoperatively shown, sublobar resection should be converted to standard lobectomy with systematic lymph node dissection.

One of the limitations of this multicenter study using PET is the wide variation in SUV among institutions. Many factors such as preparation procedures, scan acquisition, image reconstruction, and data analysis can affect SUV. In this study, we used an anthropomorphic body phantom to minimize interinstitutional variability in SUV. When performing subgroup analyses of DFS from each institution, DFS of patients with a solid tumor size of less than $0.8 \mathrm{~cm}$ or an SUVmax of less than 1.5 was significantly better than that of patients with a solid tumor size of $0.8 \mathrm{~cm}$ or more and an SUVmax of 1.5 or more (data not shown). Moreover, when using the original SUVmax values, multivariate logistic regression analysis showed that SUVmax was an independent predictor of lymph node metastasis, and DFS of patients with a solid tumor size of less than $0.8 \mathrm{~cm}$ or an SUVmax of less than 1.5 was significantly better than that of those with a solid tumor size of $0.8 \mathrm{~cm}$ or more and an SUVmax of 1.5 or more (data not shown). These findings are important to other institutions that may use our criteria of $\mathrm{pNO}$.

This study had several limitations. Because the follow-up period was short, long-term follow-up is needed to confirm 
the results of DFS. In addition, it is sometimes difficult to measure solid tumor size, especially with a large GGO component. Because this was a retrospective study, a validation cohort study is required to confirm our pN0 predictive criteria of solid tumor size on HRCT and SUVmax on FDG$\mathrm{PET} / \mathrm{CT}$.

\section{CONCLUSIONS}

Preoperative solid tumor size on HRCT and SUVmax on FDG-PET/CT are useful for prediction of pNO in clinical stage IA lung adenocarcinoma. The $\mathrm{pN} 0$ predictive criteria of solid tumor size less than $0.8 \mathrm{~cm}$ or SUVmax less than 1.5 may be helpful for avoiding systematic lymphadenectomy in patients with clinical stage IA lung adenocarcinoma.

\section{References}

1. Okada M, Koike T, Higashiyama M, Yamato Y, Kodama K, Tsubota N. Radical sublobar resection for small-sized non-small cell lung cancer: a multicenter study. J Thorac Cardiovasc Surg. 2006;132:769-75.

2. Nakayama H, Yamada K, Saito H, Oshita F, Ito H, Kameda Y, et al. Sublobar resection for patients with peripheral small adenocarcinomas of the lung: surgical outcome is associated with features on computed tomographic imaging. Ann Thorac Surg. 2007;84:1675-9.

3. National Lung Screening Trial Research Team, Aberle DR, Adams AM, Berg CD, Black WC, Clapp JD, et al. Reduced lung-cancer mortality with low-dose computed tomographic screening. N Engl J Med. 2011;365:395-409.

4. Okada M, Yoshikawa K, Hatta T, Tsubota N. Is segmentectomy with lymph node assessment an alternative to lobectomy for non-small cell lung cancer of $2 \mathrm{~cm}$ or smaller? Ann Thorac Surg. 2001;71:956-61.

5. Yoshikawa K, Tsubota N, Kodama K, Ayabe H, Taki T, Mori T. Prospective study of extended segmentectomy for small lung tumors: the final report. Ann Thorac Surg. 2002;73:1055-9.

6. Kodama K, Doi O, Higashiyama M, Yokouchi H. Intentional limited resection for selected patients with T1 N0 M0 non-small cell lung cancer. J Thorac Cardiovasc Surg. 1997;114:347-53.

7. Okada M, Tsutani Y, Ikeda T, Misumi K, Matsumoto K, Yoshimura M, et al. Radical hybrid video-assisted thoracic segmentectomy: long-term results of minimally invasive anatomical sublobar resection for treating lung cancer. Interact Cardiovasc Thorac Surg. 2012;14:5-11.
8. Jensik RJ, Faber LP, Milloy FJ, Monson DO. Segmental resection for lung carcinoma: a fifteen-year experience. J Thorac Cardiovasc Surg. 1973;66:563-72.

9. Tsutani Y, Miyata Y, Nakayama H, Okumura S, Adachi S, Yoshimura M, et al. Prognostic significance of using solid versus whole tumor size on highresolution computed tomography for predicting the pathological malignant grade of tumors in clinical stage IA lung adenocarcinoma: a multicenter study. $J$ Thorac Cardiovasc Surg. 2012;143:607-12.

10. Okada M, Nakayama H, Okumura S, Daisaki H, Adachi S, Yoshimura M, et al. Multicenter analysis of high-resolution computed tomography and positron emission tomography/computed tomography findings to choose therapeutic strategies for clinical stage IA lung adenocarcinoma. J Thorac Cardiovasc Surg. 2011;141:1384-91.

11. Goldstraw P, Crowley J, Chansky K, Giroux DJ, Groome PA, Rami-Porta R, et al. International Association for the Study of Lung Cancer International Staging Committee; Participating Institutions. The IASLC Lung Cancer Staging Project: proposals for the revision of the TNM stage groupings in the forthcoming (seventh) edition of the TNM Classification of Malignant Tumours. J Thorac Oncol. 2007;2:706-14.

12. Suzuki K, Koike T, Asakawa T, Kusumoto M, Asamura H, Nagai K, et al. A prospective radiological study of thin-section computed tomography to predict pathological noninvasiveness in peripheral clinical IA lung cancer (Japan Clinical Oncology Group 0201). J Thorac Oncol. 2011;6:751-6.

13. Delbeke D, Coleman RE, Guiberteau MJ, Brown ML, Royal HD, Siegel BA, et al. Procedure guideline for tumor imaging with 18F-FDG PET/CT 1.0. $J$ Nucl Med. 2006;47:885-95.

14. Nakayama H, Okumura S, Daisaki H, Kato Y, Uehara H, Adachi S, et al. Value of integrated positron emission tomography revised using a phantom study to evaluate malignancy grade of lung adenocarcinoma. Cancer. 2010;116:3170-7.

15. Ginsberg RH, Rubinstein LV. Randomized trial of lobectomy versus limited resection for T1N0 non-small cell lung cancer. Lung Cancer Study Group. Ann Thorac Surg. 1995;60:615-23.

16. Okada M, Tauchi S, Iwanaga K, Mimura T, Kitamura Y, Watanabe H, et al Associations among bronchioloalveolar carcinoma components, positron emission tomographic and computed tomographic findings, and malignant behavior in small lung adenocarcinomas. J Thorac Cardiovasc Surg. 2007;133: 1448-54.

17. Tsutani Y, Miyata Y, Misumi K, Ikeda T, Mimura T, Hihara J, et al. Difference in prognostic significance of maximum standardized uptake value on [18F]-fluoro2-deoxyglucose positron emission tomography between adenocarcinoma and squamous cell carcinoma of the lung. Jpn J Clin Oncol. 2011;41:890-6.

18. Nakamura K, Saji H, Nakajima R, Okada M, Asamura H, Shibata T, et al. A phase III randomized trial of lobectomy versus limited resection for small-sized peripheral non-small cell lung cancer (JCOG0802/WJOG4607L). Jpn J Clin Oncol. 2010;40:271-4. 